\title{
Long non-coding RNA CASC9 promotes tumor growth and metastasis via modulating FZD6/Wnt/ $\beta$-catenin signaling pathway in bladder cancer
}

Yonghao Zhan ${ }^{1,2}$ D, Lianghao Zhang ${ }^{1}$, Shuanbao $\mathrm{Yu}^{1}$, Jianguo Wen ${ }^{1 *}$, Yuchen Liu ${ }^{3^{*}}$ and Xuepei Zhang ${ }^{1,2^{*}}$

\begin{abstract}
Background: Accumulating evidence have highlighted the importance of long noncoding RNAs (IncRNAs) in multiple cancers development and progression. Cancer susceptibility candidate 9 (CASC9) is a novel long noncoding RNA and plays important regulatory role in diverse biological processes of cancers. However, the clinical significance and molecular mechanism of CASC9 in bladder cancer is still unknown.

Methods: Comprehensive IncRNAs profiling analysis were conducted to identify IncRNAs profile alterations and uncover valuable IncRNA candidates for bladder cancer. The expression level of CASC9 was determined in a total of 106 patients with bladder cancer. Loss-of-function experiments were performed to identify the functions of CASC9 in tumor growth and metastasis of bladder cancer in vitro and in vivo. Bioinformatics analysis and further experiments were performed to explore the molecular mechanisms underlying the functions of CASC9.

Results: This study found that CASC9 expression was markedly upregulated in bladder cancer and related to histological grade, TNM stage and prognosis. Knockdown of CASC9 inhibited tumor growth and metastasis of bladder cancer in vitro and in vivo. Mechanistically, we found that CASC9 functions as a miRNA sponge to positively regulate FZD6 expression and subsequently activates Wnt/ $\beta$-catenin signaling pathway, thus playing an oncogenic role in bladder cancer pathogenesis.

Conclusion: In summary, IncRNA CASC9 plays a critical regulatory role in bladder cancer. The CASC9/miR-497$5 \mathrm{p} /$ FZD6 axis provides insights for regulatory mechanism of bladder cancer, and new strategies for clinical practice.
\end{abstract}

Keywords: Bladder cancer, CASC9, FZD6, IncRNA, miR-497-5p

\footnotetext{
*Correspondence: wenjg@hotmail.com; liuyuchenmdcg@163.com; zhangxuepei@263.net

'Department of Urology, The First Affiliated Hospital of Zhengzhou University, No. 1 Jianshe East Road, Zhengzhou 450052, China

${ }^{3}$ Shenzhen Second People's Hospital, The First Affiliated Hospital of Shenzhen University, Health Science Center, Shenzhen 518035, China

Full list of author information is available at the end of the article
} 


\section{Background}

Bladder cancer (BC) is the sixth most frequent malignancy in males and represents one of the most common genitourinary malignancies all over the world $[1,2]$. The cause of $\mathrm{BC}$ is still unknown and the prognosis of patients with $\mathrm{BC}$ is poor under conventional treatments $[3$, 4]. Thus, the effective prognostic and therapeutic targets for $\mathrm{BC}$ are urgently needed.

The long non-coding RNAs (lncRNAs) are important new members of the ncRNA family with greater than 200 nucleotides in length, and lacking of protein coding probability $[5,6]$. Over the past decades, the rapid development of RNA genomics has highlighted the important role of lncRNAs in many human diseases, especially in cancers [7-10]. Recently, a growing number of evidence has showed that many lncRNAs play important regulatory roles in diverse biological processes of $\mathrm{BC}$, such as BLACAT2, LNMAT1, LNMAT2, PTENP1, UCA-1, HOTAIR, and etc. [11-18]. Cancer susceptibility candidate 9 (CASC9, Gene ID 101805492 in NCBI records) is a novel identified lncRNA located at 8q21.11 [19]. Recently, CASC9 originally was identified as a potential biomarker and was involved in the development and progression of multiple cancers [20-25]. For example, CASC9 forms a complex with HNRNPL and coregulates target genes expression and subsequently activates AKT-signaling pathway, in hepatocellular carcinoma. CASC9 activates LAMC2 expression through altering LAMC2 promoter H3K27me3 level by recruits $\mathrm{CBP}$ and subsequently promotes esophageal squamous cell carcinoma metastasis. CASC9 also recruiting EZH2 to PDCD4 promoter and alters H3K27me3 level and subsequently promotes esophageal squamous cell carcinoma metastasis by activating PDCD4 expression [24]. However, the biological function and underlying mechanism of action of CASC9 in BC is completely unknown.

In the present study, we performed comprehensive lncRNAs profiling analyses to identify lncRNAs profile alterations and uncover valuable lncRNA candidates for BC using The Cancer Genome Atlas (TCGA-BLCA) data and Gene Expression Omnibus (GEO) data. We found that CASC9 was significantly up-regulated in BC and positively correlated with poor prognosis. Meanwhile, CASC9 expression was markedly upregulated in $\mathrm{BC}$ and related to histological grade, TNM stage and prognosis. Furthermore, further experiments demonstrated that knockdown of CASC9 inhibited growth and metastasis of bladder cancer cells (BCCs) in vitro and in vivo. Mechanistically, we found CASC9 was mainly distributed in the cytoplasm and knockdown of CASC9 increased miR-497-5p expression and subsequently inhibited FZD6 expression, in a ceRNA-dependent way. Moreover, knockdown of miR-497-5p reversed FZD6 expression inhibition and reversed malignant phenotypes inhibition of BCCs. Together, our results suggest that CASC9 is a powerful tumor biomarker, which highlight its potential clinical utility as a promising diagnostic and therapeutic target of $\mathrm{BC}$.

\section{Materials and methods \\ Data extraction and bioinformatics analysis}

Gene expression profiles was downloaded from The Cancer Genome Atlas (TCGA-BLCA) database and the Gene Expression Omnibus (GSE89006) database. The edgeR package was used to analysis differentially expressed lncRNA in TCGA-BLCA detasets. The limma package was used to analysis differentially expressed lncRNA in GEO detasets. The GEPIA (http://gepia.cancer-pku.cn/index.html) was used to analysis prognosis of $\mathrm{BC}$ in TCGA-BLCA detasets. The IncLocator was used to predict the subcellular localization of CASC9.

\section{Clinical sample collection and cell culture}

Clinical sample collection and cell culture were performed following standard protocols as previously reported [18]. Clinicopathological features of patients was shown in Supplementary Table 1. The normal urothelial cell line and $\mathrm{BC}$ cell lines were purchased from the Institute of Cell Research, Chinese Academy of Sciences, Shanghai, China.

\section{Quantitative real-time PCR and western blotting analysis} Quantitative real-time PCR and western blotting analysis were performed following standard protocols as previously reported [18]. The detailed primer sequences included in this study are shown in Supplementary Table 2. The detailed protocols included in this study are also shown in Supplementary materials and methods. The primary antibody FZD6/Ki67/MMP14 and secondary antibody were purchased from Abcam, Hong Kong, China. The primary antibody $\beta$-catenin/E-cadherin $/ \mathrm{N}$ cadherin/vimentin/slug were purchased from Cell Signaling Technology, USA.

\section{Cell proliferation and cell metastasis assays}

Cell Counting Kit-8 (CCK-8) and ethynyl-2-deoxyuridine (EdU)-incorporation assay were used to determine $\mathrm{BC}$ cell proliferation. Wound-healing assays and transwell assays were used to determine the migratory abilities and invasive abilities of BCCs, respectively. Cell Counting Kit- 8 (CCK-8), ethynyl-2-deoxyuridine (EdU)-incorporation assay, wound-healing assays and transwell assays were performed following standard protocols as previously reported [16]. The detailed protocols included in this study are also shown in Supplementary materials and methods. 


\section{Mouse model experiments}

All animal experiments were approved by the Institutional Animal Care and Use Committee (IACUC) of The First Affiliated Hospital of Zhengzhou University and conducted in accordance with its recommendations and ethical regulations. For the tumour xenograft implantation experiment, $1 \times 10^{6} \mathrm{SW780}$ cells were injected subcutaneously into 5-week-old male BALB/c nude mice (Vital River, Beijing, China), which were subsequently sacrificed 5 weeks later. For the metastasis experiment, $1 \times 10^{5}$ 5637-Luc cells were suspended in $200 \mu \mathrm{L}$ PBS and injected into the lateral tail veins of 5 -week-old male B-NDG mice (BIOCYTOGEN, Beijing, China). Four weeks later, mice were anaesthetized with isoflurane and D-luciferin sodium salt $(150 \mathrm{mg} / \mathrm{kg})$ was injected intraperitoneally, and cancer cells were detected with an in vivo imaging system, Xenogen IVIS (PerkinElmer, MA, USA). The total flux in photons per second was calculated and reported for each mouse's lung and liver region using Living Image 4.3.1 (PerkinElmer/Caliper).

FISH, immunohistochemistry and immunofluorescence RNA fluorescent in situ hybridization (FISH), immunohistochemistry and immunostaining were performed following standard protocols as previously reported [16]. CASC9 and U6 probes were designed and synthesized by Ribobio Company and labeled with Cy3 fluorescent dye. The primary antibody FZD6/Ki67 were purchased from Abcam, Hong Kong, China. The primary antibody E-cadherin/Ncadherin and fluorescence secondary antibody were purchased from Cell Signaling Technology, USA.

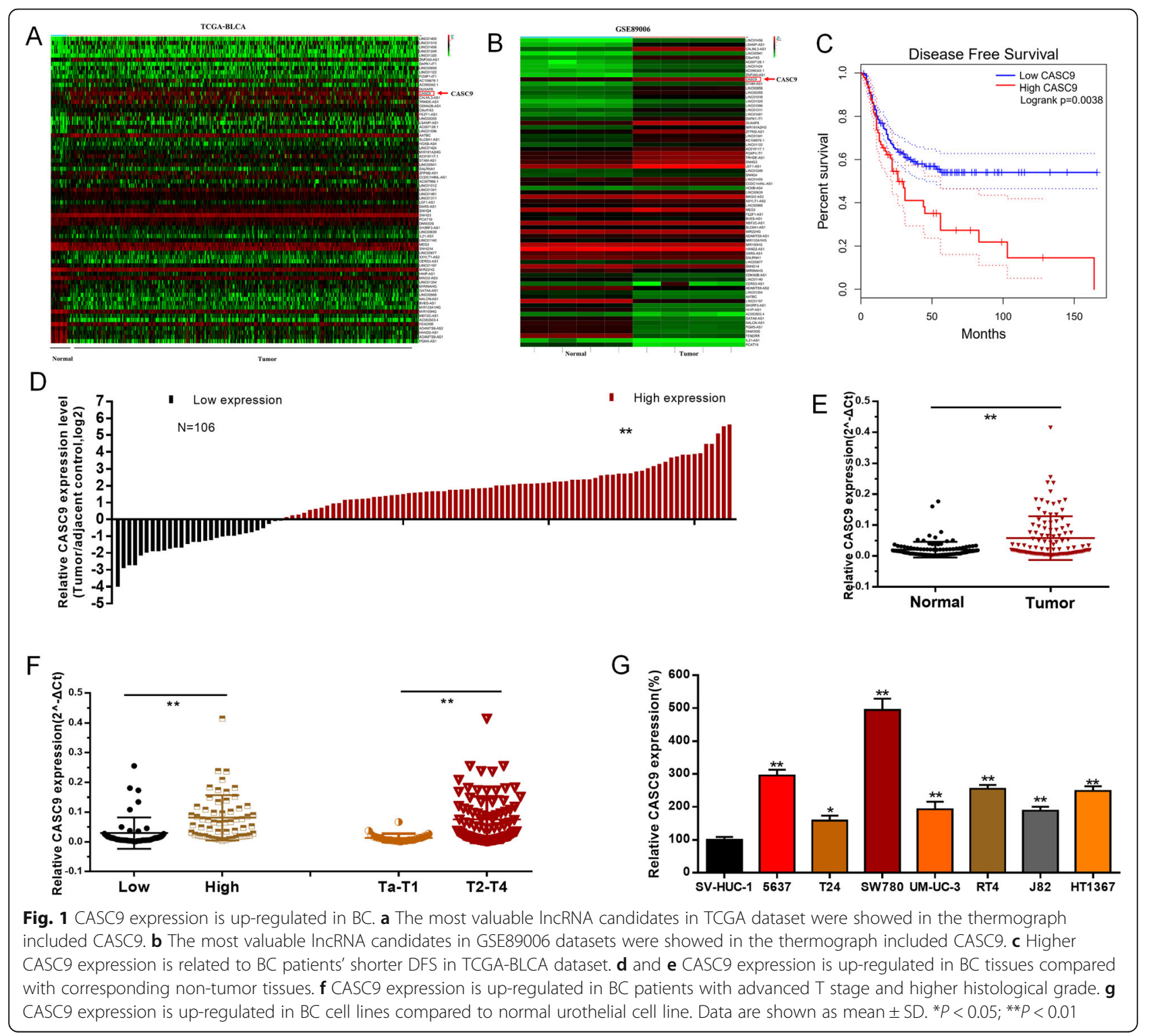




\section{Statistical analyses}

Quantitative data from three independent experiments were analyzed by Student's t-test or $\mathrm{x} 2$ test and results were expressed as mean \pm SD. Kaplan-Meier survival analysis was used to evaluate the cumulative survival probability. $P<0.05$ were considered statistically significant. All statistical tests were conducted using SPSS 19.0 (SPSS Inc. Chicago, IL, USA).

\section{Results}

\section{CASC9 expression is up-regulated in BC}

We conducted comprehensive lncRNAs expression profile analyses using TCGA-BLCA dataset and a GEO detaset (GSE89006) to identify lncRNAs profile alterations and uncover valuable lncRNA candidates for BC and explored their clinical relevance. We found CASC9 expression were up-regulated in TCGA-BLCA (Fig. 1a and Fig. S1A) and GSE89006 (Fig. 1b and Fig. S1B) datasets and higher CASC9 expression was associated with BC patients' shorter DFS (Fig. 1c) in TCGA-BLCA dataset. The relative expression level of CASC9 was determined by qRT-PCR in BC cohort data to confirm the result of bioinformatics analysis. Meanwhile, CASC9 expression was up-regulated in $\mathrm{BC}$ tissues compared to paired non-tumor tissues (Fig. 1d and e) and higher CASC9 expression was positively correlated with advanced $\mathrm{T}$ stage and higher histological grade (Fig. 1f). Moreover, CASC9 expression was elevated in BC cell lines compared to SV-HUC-1 (Fig. 1g). The CASC9 specific shRNAs markedly down-regulated CASC9 expression in BC cells (Fig. S1C) and the CASC9 vector significantly increased CASC9 expression in BC cells
(Fig. S1D). Correlation between CASC9 expression and clinicopathological features of $\mathrm{BC}$ patients was shown in Table 1.

\section{CASC9 promotes the proliferation of bladder cancer cells}

To investigate whether CASC9 regulates cell proliferation of $\mathrm{BC}$, we conducted CCK- 8 assay and Edu assay to determine the proliferation changes of BCCs. Our results showed that knockdown of CASC9 inhibited the proliferation of BCCs (Fig. 2a and d) and overexpressing CASC9 promoted the proliferation of BCCs (Fig. $2 b$ and c) in vitro. Meanwhile, we performed generation of xenograft to investigate the biological function and regulatory mechanism of CASC9 in vivo. Our result showed knockdown of CASC9 decreased tumor growth and tumor weight (Fig. 2e-g), and increased tumor free survival time (Fig. S2A). Moreover, we found knockdown of CASC9 decreased Ki67 expression (Fig. 2h) of BCCs in vivo. These results indicated that CASC9 promotes the proliferation of bladder cancer cells.

\section{CASC9 does not affect the apoptosis of bladder cancer cells} We further determined whether CASC9 regulates cell apoptosis of BCCs. The changes in bladder cancer cell apoptosis were determined using flow cytometry. Regrettably, there was no difference in the apoptosis of BCCs transfected with the corresponding specific shRNA (Fig. $2 \mathrm{i})$ and overexpression vector (Fig. 2j). The results indicated that CASC9 does not affect the apoptosis of bladder cancer cells.

Table 1 Correlation between CASC9 expression and clinicopathological features of UCB patients

\begin{tabular}{|c|c|c|c|c|c|}
\hline \multirow[t]{2}{*}{ Parameters Total } & \multirow[t]{2}{*}{ Group } & \multirow[t]{2}{*}{ Total } & \multicolumn{2}{|c|}{ CASC9 expression } & \multirow[t]{2}{*}{$P$ value } \\
\hline & & & High & Low & \\
\hline \multirow[t]{2}{*}{ Gender } & Male & 79 (75\%) & $58(55 \%)$ & $21(20 \%)$ & 0.759 \\
\hline & Female & $27(25 \%)$ & $19(18 \%)$ & $8(8 \%)$ & \\
\hline \multirow[t]{2}{*}{ Age (years) } & $<60$ & $37(35 \%)$ & $27(25 \%)$ & $10(9 \%)$ & 0.956 \\
\hline & $\geq 60$ & $69(65 \%)$ & $50(47 \%)$ & $19(18 \%)$ & \\
\hline \multirow[t]{2}{*}{ Tumor size $(\mathrm{cm})$} & $<3 \mathrm{~cm}$ & $42(40 \%)$ & $27(25 \%)$ & $15(14 \%)$ & 0.118 \\
\hline & $\geq 3 \mathrm{~cm}$ & $64(60 \%)$ & $50(47 \%)$ & $14(13 \%)$ & \\
\hline \multirow[t]{2}{*}{ Multiplicity } & Single & $59(56 \%)$ & $38(36 \%)$ & $21(20 \%)$ & $0.033 *$ \\
\hline & Multiple & 47 (44\%) & 39 (37\%) & $8(8 \%)$ & \\
\hline \multirow[t]{2}{*}{ Histological grade } & L & $48(45 \%)$ & $25(24 \%)$ & $23(22 \%)$ & $0.001 * *$ \\
\hline & $\mathrm{H}$ & $58(55 \%)$ & $52(49 \%)$ & $6(6 \%)$ & \\
\hline \multirow[t]{2}{*}{ Tumor stage $T$} & $\mathrm{Ta}, \mathrm{T} 1$ & $26(25 \%)$ & $10(9 \%)$ & $16(15 \%)$ & $0.001 * *$ \\
\hline & $\mathrm{T} 2-\mathrm{T} 4$ & $80(75 \%)$ & $67(63 \%)$ & $13(12 \%)$ & \\
\hline \multirow[t]{2}{*}{ Lymph nodes metastasis } & NO & $92(87 \%)$ & $65(61 \%)$ & $27(25 \%)$ & 0.153 \\
\hline & YES & $14(13 \%)$ & $13(12 \%)$ & $1(1 \%)$ & \\
\hline
\end{tabular}

${ }^{*} P<0.05 ;{ }^{* *} P<0.01 . P<0.05$ was considered significant (Chi-square test between 2 groups) 


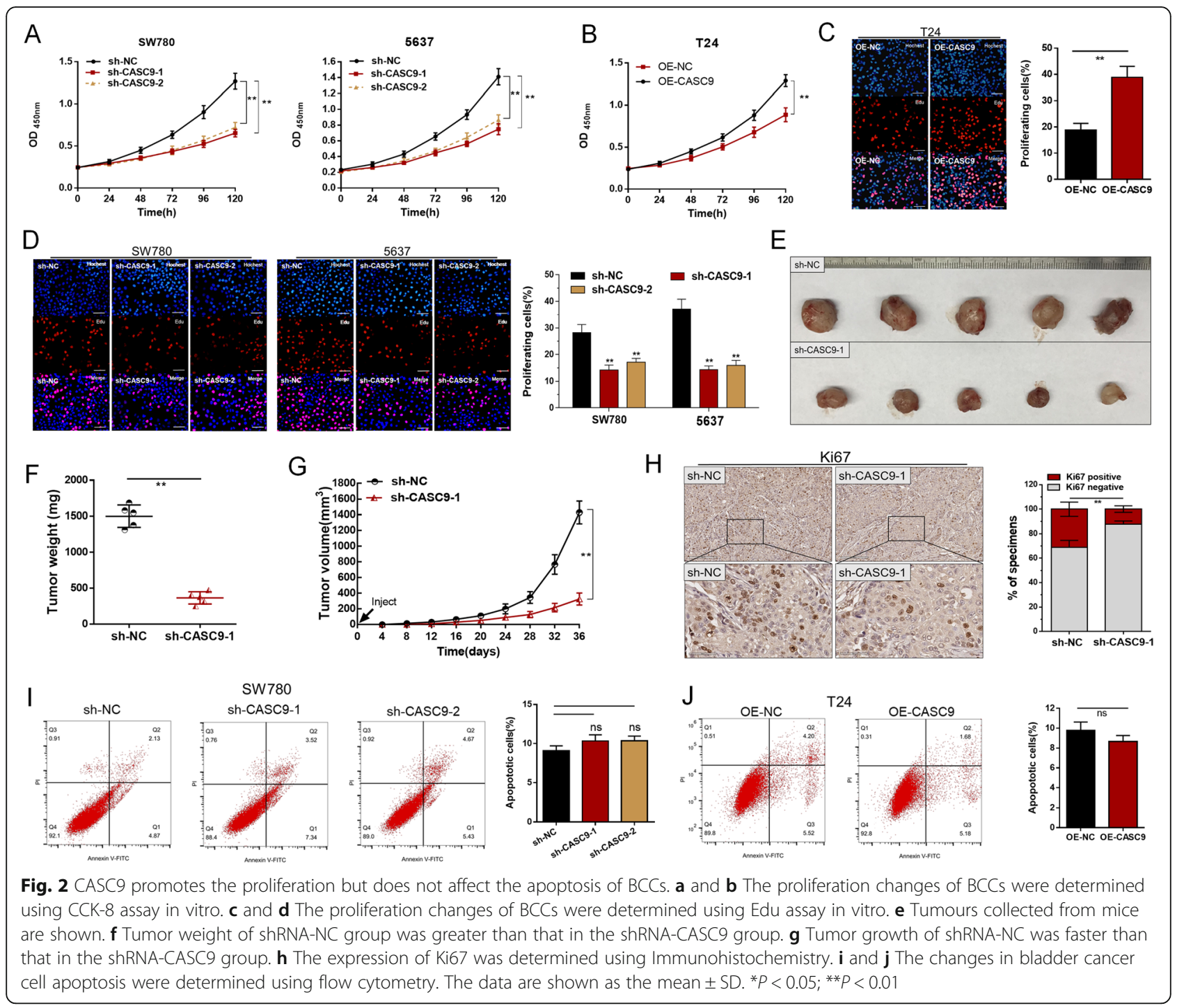

\section{CASC9 promotes the migration and invasion of bladder cancer cells}

We further determined whether CASC9 regulates the migration and invasion of BCCs using wound healing assay and transwell assay, respectively. We found that knockdown of CASC9 inhibited migration (Fig. 3a) and invasion of BCCs (Fig. 3b). Meanwhile, we found overexpressing CASC9 promoted migration (Fig. 3c) and invasion (Fig. 3d) of BCCs. To investigate the biological function and regulatory mechanism of CASC9 in vivo, we also performed whole-body fluorescent imaging system. Our result showed knockdown of CASC9 decreased luciferase signals (Fig. 3e) but had no effect on mouse weight (Fig. S2C). The hematoxylin-eosin staining was performed to the lung tissue to observed the metastases in the groups (Fig. 3f), respectively. Our results showed knockdown of CASC9 significantly reduced the number of pulmonary metastases and metastases size (Fig. 3f). Moreover, further results showed knockdown of CASC9 decreased Ki67 (Fig. 3g) expression of BCCs in vivo. These results indicated that CASC9 promotes the migration and invasion of bladder cancer cells.

\section{CASC9 positively regulates the expression of FZD6}

Through comprehensive transcriptional analysis using TCGA dataset and our dataset, we found that CASC9 expression were positively correlated with FZD6 expression in BC (Fig. 4a). Furthermore, FZD6 expression are up-regulated in $\mathrm{BC}$ (Fig. S3A) and FZD6 expression is related to the OS and DFS of $\mathrm{BC}$ patients in TCGA-BLCA dataset (Fig. S3B). Correlation between FZD6 expression and clinicopathological features of BC patients was shown in Table 2. Our result showed knockdown of CASC9 decreased FZD6, $\beta$-catenin expression and inhibited EMT of BCCs 


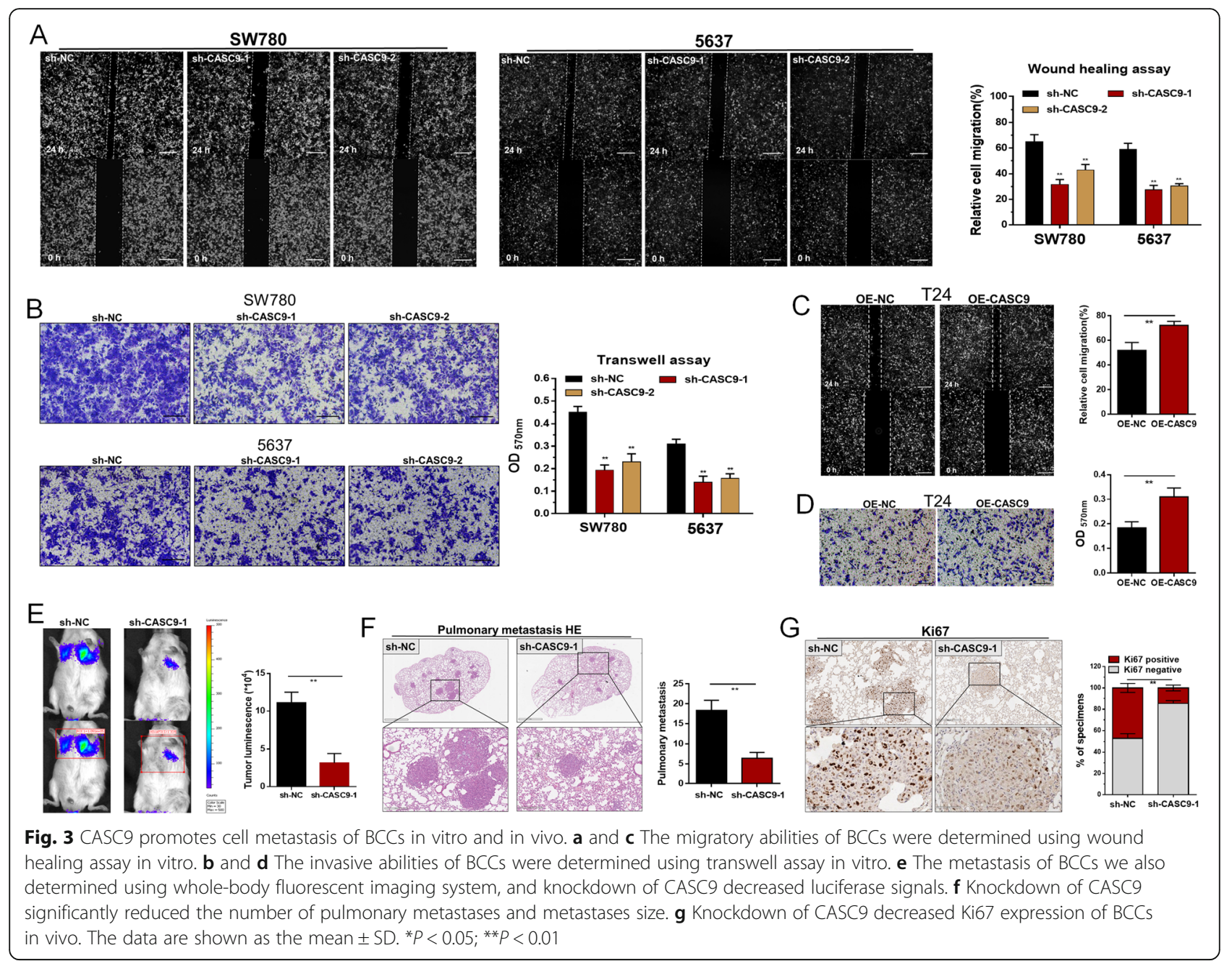

(Fig. 4b-d) in vitro. Meanwhile, we found knockdown of CASC9 decreased FZD6 (Fig. 4e-g) and inhibited EMT (Fig. S2B and D) of bladder cancer cells in vivo. Moreover, CASC9 and FZD6 were co-expressed in bladder cancer cells (Fig. 4g). These results indicated that CASC9 may activates Wnt/ $\beta$-catenin signaling pathway via regulating FZD6 expression and subsequently promotes growth and metastasis of BCCs.

\section{CASC9 regulates FZD6 expression via sponging miR-497-5p}

To explore the regulatory mechanism of CASC9 on FZD6, we predicted the subcellular localization of CASC9 using lncLocator (Fig. 5a), and performed RNAFISH (Fig. 5b) and qRT-PCR (Fig. 5c) to verify the result in BCCs. The results revealed that CASC9 was distributed mostly in cytoplasm of BCCs. To elucidate whether CASC9 functioned as a ceRNA in BCCs, we used RegRNA 2.0 and Targetsacn 7.1 to predict potential shared target microRNA of CASC9 and FZD6. The results revealed that CASC9 and FZD6 have shared putative binding sites with miR-497-5p cluster (Fig. 5d).
Furthermore, further experimental results showed knockdown of CASC9 increased miR-497-5p expression (Fig. 5e) and elevated miR-497-5p decreased FZD6 expression in BCCs (Fig. 5f). Meanwhile, dual-luciferase reporter assay showed miR-497-5p inhibited the luciferase activity in CASC9-Wt and FZD6-Wt group, with no effect in CASC9-Mut and FZD6-Mut group (Fig. 5g and h). Knockdown of CASC9 decreased the luciferase activity in FZD6-Wt group (Fig. 5i). These results indicated that CASC9 positively regulates FZD6 expression via sponging miR-497-5p in BCCs.

\section{Knockdown of miR-497-5p reverses tumor growth and metastasis inhibited by silencing CASC9}

To validate the regulatory mechanism of the CASC9/ miR-497-5p/FZD6 axis, we further performed miR-497$5 \mathrm{p}$ blocking experiments. Our results showed that knockdown of miR-497-5p significantly reversed the proliferation (Fig. 6a), migration (Fig. 6d) and invasion (Fig. 6e) of shRNA-CASC9 group in vitro. Meanwhile, knockdown of miR-497-5p reversed tumor growth of 


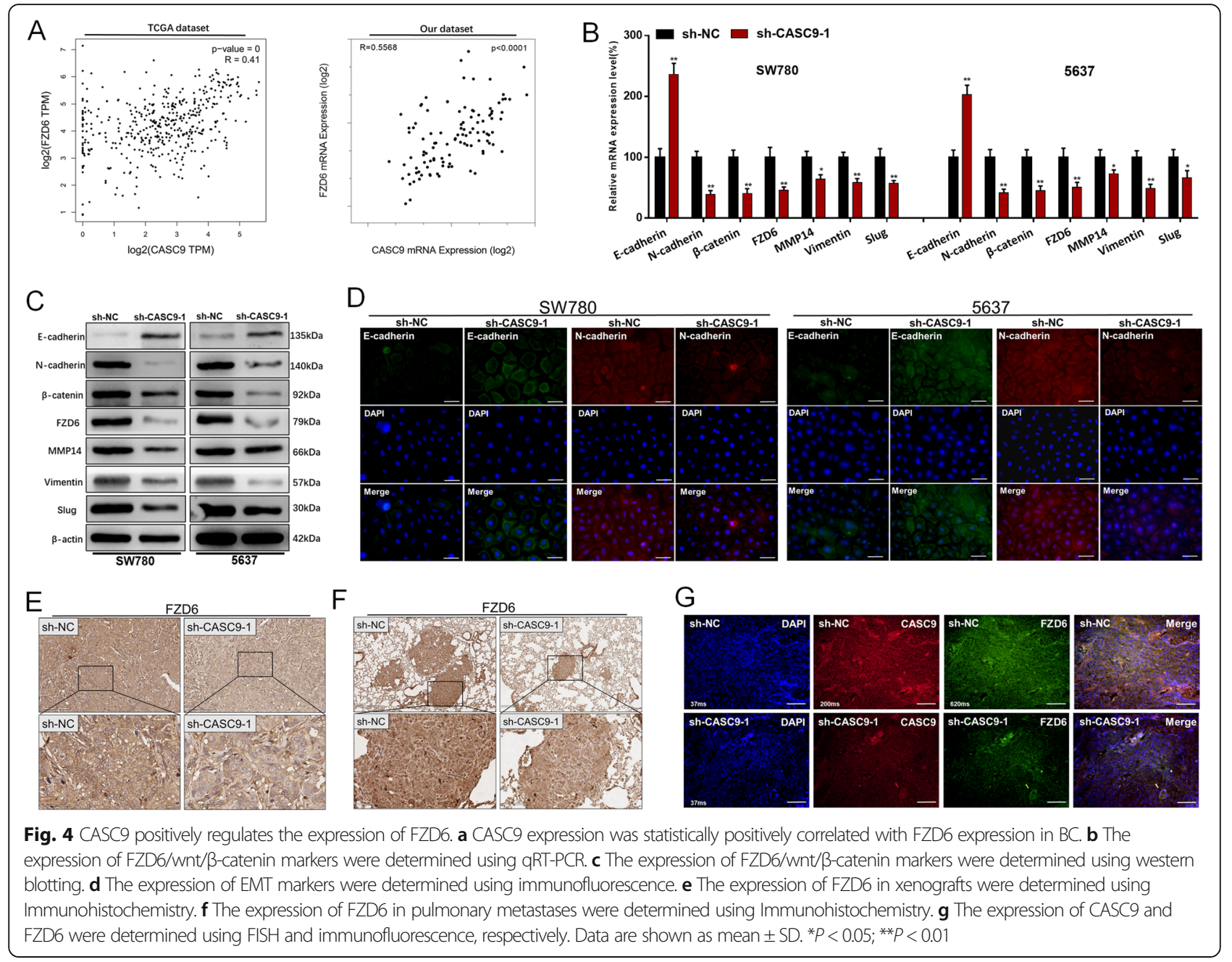

Table 2 Correlation between FZD6 expression and clinicopathological features of UCB patients

\begin{tabular}{|c|c|c|c|c|c|}
\hline \multirow[t]{2}{*}{ Parameters Total } & \multirow[t]{2}{*}{ Group } & \multirow[t]{2}{*}{ Total } & \multicolumn{2}{|c|}{ FZD6 expression } & \multirow[t]{2}{*}{$P$ value } \\
\hline & & & High & Low & \\
\hline \multirow[t]{2}{*}{ Gender } & Male & 79 (75\%) & $56(53 \%)$ & $23(22 \%)$ & 0.682 \\
\hline & Female & $27(25 \%)$ & $18(17 \%)$ & $9(8 \%)$ & \\
\hline \multirow[t]{2}{*}{ Age (years) } & $<60$ & $37(35 \%)$ & $25(24 \%)$ & $12(11 \%)$ & 0.713 \\
\hline & $\geq 60$ & $69(65 \%)$ & $49(46 \%)$ & $20(19 \%)$ & \\
\hline \multirow[t]{2}{*}{ Tumor size $(\mathrm{cm})$} & $<3 \mathrm{~cm}$ & $42(40 \%)$ & $24(23 \%)$ & $18(17 \%)$ & $0.021 *$ \\
\hline & $\geq 3 \mathrm{~cm}$ & $64(60 \%)$ & $50(47 \%)$ & $14(13 \%)$ & \\
\hline \multirow[t]{2}{*}{ Multiplicity } & Single & $59(56 \%)$ & $40(38 \%)$ & $19(18 \%)$ & 0.613 \\
\hline & Multiple & 47 (44\%) & $34(32 \%)$ & $13(12 \%)$ & \\
\hline \multirow[t]{2}{*}{ Histological grade } & $\mathrm{L}$ & $48(45 \%)$ & $26(25 \%)$ & $22(21 \%)$ & $0.003 * *$ \\
\hline & $\mathrm{H}$ & $58(55 \%)$ & $48(45 \%)$ & $10(9 \%)$ & \\
\hline \multirow[t]{2}{*}{ Tumor stage $T$} & $\mathrm{Ta}, \mathrm{T1}$ & $26(25 \%)$ & $11(10 \%)$ & $15(14 \%)$ & $0.001 * *$ \\
\hline & $\mathrm{T} 2-\mathrm{T} 4$ & $80(75 \%)$ & $63(59 \%)$ & $17(16 \%)$ & \\
\hline \multirow[t]{2}{*}{ Lymph nodes metastasis } & NO & $92(87 \%)$ & $63(59 \%)$ & $29(27 \%)$ & 0.650 \\
\hline & YES & $14(13 \%)$ & $11(10 \%)$ & $3(3 \%)$ & \\
\hline
\end{tabular}

${ }^{*} P<0.05 ;{ }^{*} P<0.01 . P<0.05$ was considered significant (Chi-square test between 2 groups) 

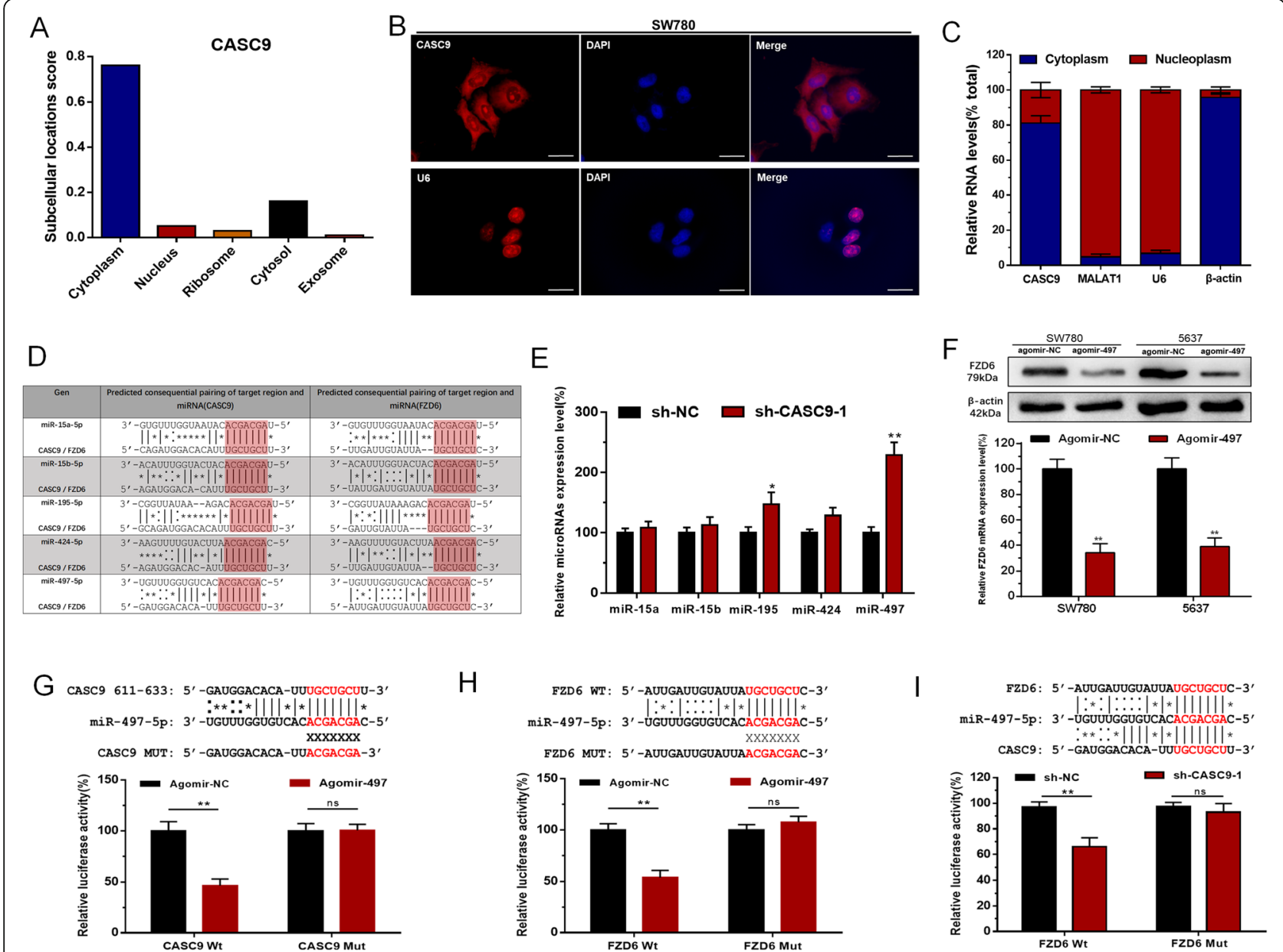

Fig. 5 CASC9 positively regulates FZD6 expression via sponging miR-497-5p. a IncLocator results revealed that CASC9 was distributed mostly in the cytoplasm. b The RNA-FISH results revealed that CASC9 was distributed mostly in the cytoplasm of BCCs. c Subcellular localization of CASC9 and control genes analyzed with quantitative RT-PCR in biochemically fractionated SW780 cells. $\mathbf{d}$ The bio-information analysis results showed CASC9 and FZD6 have common putative binding sites with miR-497-5p cluster. e Knockdown of CASC9 increased miR-497-5p expression in BCCs. f Overexpressing miR-497-5p decreased FZD6 expression in BCCs. g CASC9 have putative binding sites with miR-497-5p and agomir-497 significantly inhibited luciferase activity of CASC9-Wt group. $\mathbf{h}$ The 3'UTR sequence of FZD6 is complementary to the seed sequence of miR-497$5 p$ and agomir-497 significantly inhibited luciferase activity of FZD6-Wt group. i Knockdown of CASC9 decreased the luciferase activity of BCCS transfected with FZD6-Wt. Data are shown as mean \pm SD. ${ }^{*} P<0.05 ;{ }^{* *} P<0.01$

shRNA-CASC9 group (Fig. 6b and c) in vivo. Moreover, knockdown of miR-497-5p significantly reversed FZD6 (Fig. 6f) and Ki67 (Fig. 6g) expression in BCCs. These results indicated that CASC9 promotes malignant phenotypes of BCCs through positively regulating FZD6 expression via miR-497-5p-dependent manner. As shown in Fig. 6h, CASC9 functions as a miRNA sponge to positively regulate FZD6 expression through sponging miR-497-5p and subsequently activates $\mathrm{Wnt} / \beta$-catenin signaling pathway.

\section{Discussion}

Bladder cancer is the most common and most aggressive malignant primary adult genitourinary tumor [26, 27]. Despite improvements in current clinical treatment such as surgery, radiation therapy, and chemotherapy, the overall survival (OS) time of $\mathrm{BC}$ patients has not been significantly improved [28-32]. So novel markers for diagnosis at early stage and more efficient and safer therapeutic method are urgently needed.

The lncRNAs are longer than 200 nucleotides, important members of no-coding RNA family, which have no ability to encode proteins. Recently, the rapid development of RNA genomics has uncovered lncRNAs play key roles in a wide variety of human diseases including cancers, through regulating gene expression at different processing levels, including chromatin modification, transcription and posttranscriptional regulation [33, 34]. For example, LncRNA LNMAT1 could recruit hnRNPL to CCL2 promoter and subsequently epigenetically 


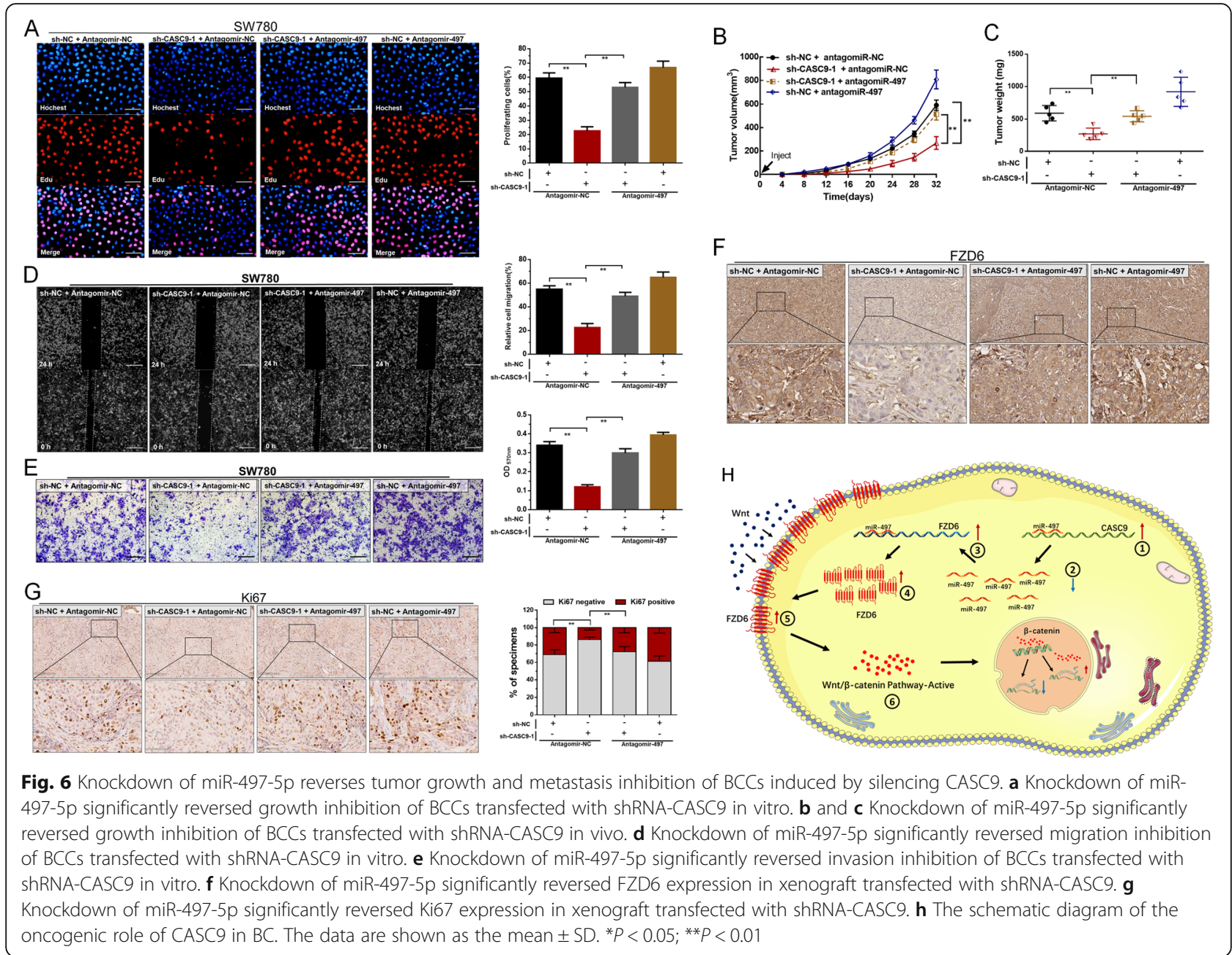

activate CCL2 expression in BC [12]. LncRNA LUCAT1 could interact with PTBP1 and subsequently facilitate altered alternative splicing of DNA damage related genes in colorectal cancer [35]. LncRNA XIST have also been reported to down-regulate the expression of miR-21 as a miRNA sponge and subsequently increase the expression of IL-12A in primary graft dysfunction [36]. LncRNAs can be distributed in the cytoplasm, nucleus and other cellular substructures, and lncRNAs subcellular localization are closely related to their biology function and molecular roles $[37,38]$. CASC9 is a new identified lncRNA located on human chromosome 8 (8q21.11) [39]. Recently, CASC9 originally was identified as a key regulator and the differentially expressed CASC9 have become highlighted in some somatic cancers $[25,40]$. However, the clinical significance and biological function of CASC9 in BC are completely unknown.

In the current study, we found CASC9 expression was significantly up-regulated in $\mathrm{BC}$ tissues and elevated CASC9 expression was positively correlated with higher histological grade, advanced $\mathrm{T}$ stage and poor prognosis.
Furthermore, our further experiments demonstrated that CASC9 deletion inhibited tumor growth and metastasis of $\mathrm{BC}$ in vitro and in vivo. Mechanistically, through performing comprehensive transcriptional analysis, we found that CASC9 expression was positively correlated with FZD6 expression in BC tissues. Meanwhile, FZD6 expression was also up-regulated in our dataset and knockdown of CASC9 decreased the expression of FZD6. Furthermore, a great number of studies have indicated that FZD6 is a key regulator of Wnt/ $\beta$-catenin signaling pathway and knockdown of CASC9 decreases FZD6, $\beta$-catenin expression and inhibits EMT in BCCs. To explore the regulatory mechanism of CASC9 on FZD6, we detected the subcellular localization of CASC9 using RNA-FISH. These results revealed that CASC9 is distributed mostly in the cytoplasm of BCCs, which suggest that CASC9 may play the regulatory function in posttranscriptional level. Next, through performing bioinformation analysis we found CASC9 and FZD6 have potential shared binding sites with miR-497-5p cluster and further experiments demonstrated that CASC9 
decreased miR-497-5p expression as a microRNA sponge and subsequently increased FZD6 expression. Moreover, knockdown of miR-497-5p reversed FZD6 expression and malignant phenotypes inhibition of BCCs induced by silencing CASC9.

\section{Conclusions}

In summary, our study revealed that CASC9 positively regulates FZD6 expression through sponging miR-497$5 \mathrm{p}$ and subsequently activates $\mathrm{Wnt} / \mathrm{\beta}$-catenin signaling pathway, thus playing an oncogenic role in $\mathrm{BC}$ pathogenesis. Based on our results, we propose a network of CASC9/miR-497/FZD6 as an underlying regulatory mechanism mediating occurrence and development of BC. Cumulatively, the findings of the present study suggest that CASC9 is a powerful regulator in $\mathrm{BC}$, which highlight its potential clinical utility as a promising diagnostic and therapeutic target of BC.

\section{Supplementary information}

Supplementary information accompanies this paper at https://doi.org/10. 1186/s13046-020-01624-9.

Additional file 1: Figure S1. CASC9 expression is up-regulated in BC. A: The expression of CASC9 in BC was significantly up-regulated in TCGABLCA dataset. B: The expression of CASC9 in BC was significantly upregulated in GSE89006 dataset. C: The CASC9 specific shRNAs significantly decreased CASC9 expression in SW780 and 5637. D: The CASC9 vector significantly increased CASC9 expression in T24. Data are shown as mean \pm SD. ${ }^{*} P<0.05$; ${ }^{*} P<0.01$.

Additional file 2: Figure S2. CASC9 promotes cell proliferation and metastasis of BCCs. A: Tumor free mouse proportion of shRNA-CASC9 group was higher than that in the shRNA-NC group. B: The expression of FZD6 and EMT markers in xenografts were determined using GRT-PCR. C: There was no significant difference between the mouse weight of two treatment group. D: The expression of EMT markers in pulmonary metastases were determined using Immunohistochemistry. Data are shown as mean \pm SD. ${ }^{*} P<0.05 ;{ }^{*} P<0.01$.

Additional file 3: Figure S3. FZD6 expression is up-regulated in BC. A: FZD6 expression is up-regulated in BC tissues compared with corresponding non-tumor tissues and elevated FZD6 expression is positively correlated with advanced T stage and higher histological grade. B: FZD6 expression is related to the OS and DFS of BC patients in TCGA-BLCA dataset. Data are shown as mean \pm SD. ${ }^{*} P<0.05$; ${ }^{* *} P<0.01$.

Additional file 4: Supplementary Table 1. Summary of clinicopathological features of tissues of bladder cancer

Additional file 5: Supplementary Table 2. The primer sequences included in this study.

Additional file 6. Supplemental Materials and Methods.

\section{Abbreviations}

IncRNA: Long non-coding RNA; CASC9: Cancer susceptibility candidate 9; FZD6: Frizzled Class Receptor 6; ceRNA: Competing endogenous RNA BCCs: Bladder cancer cells; OS: Overall survival; DFS: Disease free survival; qRT-PCR: Quantitative real time-PCR; EdU: Ethynyl-2-deoxyuridine; FISH: Fluorescent in situ hybridization; EMT: Epithelial-mesenchymal transition; TCGA: The Cancer Genome Atlas

\section{Acknowledgments}

The authors are indebted to all the donors whose names were not included in the author list, but who participated in our study.

\section{Authors' contributions}

ZX, LY and WJ contributed to the experiment design, manuscript draft, and data analysis. $Z Y$ and LY contributed to the experiment implementation, manuscript draft and data analysis. ZL and YS collected clinical samples. All authors have read and approved the final manuscript.

\section{Funding}

This work was supported by China Postdoctoral Science Foundation [2019 M662544], National Natural Science Foundation of China [81773257. 81972867], Henan sicence and technology project (162102310204), Natural Science Foundation of Guangdong (2018B030306023) and the Shenzhen Municipal Government of China (JCYJ20180507184642475).

Availability of data and materials

All data in our study are available upon request.

\section{Ethics approval and consent to participate}

This study was approved by the ethics committee of The First Affiliated Hospital of Zhengzhou University and The First Affiliated Hospital of Shenzhen University, and written informed consents were obtained before any operation to patients. The authors confirmed that we have obtained written consent from the patients to publish this manuscript.

\section{Consent for publication}

The authors confirmed that we have obtained written consent from the patients to publish this manuscript.

\section{Competing interests}

We declare that we have no financial and personal relationships with other people or organizations that can inappropriately influence our work.

\section{Author details}

${ }^{1}$ Department of Urology, The First Affiliated Hospital of Zhengzhou University, No. 1 Jianshe East Road, Zhengzhou 450052, China. ${ }^{2}$ Key Laboratory of Precision Diagnosis and Treatment for Chronic Kidney Disease in Henan Province, No. 1 Jianshe East Road, Zhengzhou 450052, China.

${ }^{3}$ Shenzhen Second People's Hospital, The First Affiliated Hospital of

Shenzhen University, Health Science Center, Shenzhen 518035, China.

Received: 30 January 2020 Accepted: 15 June 2020

Published online: 16 July 2020

\section{References}

1. Siegel RL, Miller KD, Jemal A. Cancer statistics, 2019. CA Cancer J Clin. 2019; 69:7-34.

2. Bray F, Ferlay J, Soerjomataram I, Siegel RL, Torre LA, Jemal A. Global cancer statistics 2018: GLOBOCAN estimates of incidence and mortality worldwide for 36 cancers in 185 countries. CA Cancer J Clin. 2018:68:394-424.

3. Fradet Y. Phenotypic characterization of bladder cancer. Eur Urol. 1998; 33(Suppl 4):5-6.

4. Oosterlinck W, Lobel B, Jakse G, Malmström PU, Stöckle M, Sternberg C. Guidelines on bladder cancer. Eur Urol. 2002;41:105-12

5. Engreitz JM, Ollikainen N, Guttman M. Long non-coding RNAs: spatial amplifiers that control nuclear structure and gene expression. Nat Rev Mol Cell Biol. 2016:17:756-70

6. Zhao XY, Lin JD. Long noncoding RNAs: a new regulatory code in metabolic control. Trends Biochem Sci. 2015:40:586-96.

7. Devaux Y, Zangrando J, Schroen B, Creemers EE, Pedrazzini T, Chang CP, Dorn GW II, Thum T, Heymans S. Long noncoding RNAs in cardiac development and ageing. Nat Rev Cardiol. 2015;12:415-25.

8. Ørom UA, Shiekhattar R. Long noncoding RNAs usher in a new era in the biology of enhancers. Cell. 2013;154:1190-3.

9. Mercer TR, Mattick JS. Structure and function of long noncoding RNAs in epigenetic regulation. Nat Struct Mol Biol. 2013;20:300-7.

10. Thum T, Condorelli G. Long noncoding RNAs and microRNAs in cardiovascular pathophysiology. Circ Res. 2015;116:751-62.

11. He W, Zhong G, Jiang N, Wang B, Fan X, Chen C, Chen X, Huang J, Lin T. Long noncoding RNA BLACAT2 promotes bladder cancer-associated lymphangiogenesis and lymphatic metastasis. J Clin Invest. 2018;128:861-75. 
12. Chen C, He W, Huang J, Wang B, Li H, Cai Q, Su F, Bi J, Liu H, Zhang B, et al. LNMAT1 promotes lymphatic metastasis of bladder cancer via CCL2 dependent macrophage recruitment. Nat Commun. 2018;9:3826.

13. Chen C, Luo Y, He W, Zhao Y, Kong Y, Liu H, Zhong G, Li Y, Li J, Huang J, et al. Exosomal long noncoding RNA LNMAT2 promotes lymphatic metastasis in bladder cancer. J Clin Invest. 2020;130:404-21.

14. Zheng R, Du M, Wang X, Xu W, Liang J, Wang W, Lv Q, Qin C, Chu H, Wang $M$, et al. Exosome-transmitted long non-coding RNA PTENP1 suppresses bladder cancer progression. Mol Cancer. 2018;17:143.

15. Xue M, Chen W, Xiang A, Wang R, Chen H, Pan J, Pang H, An H, Wang X, Hou $H$, Li X. Hypoxic exosomes facilitate bladder tumor growth and development through transferring long non-coding RNA-UCA1. Mol Cancer. 2017;16:143.

16. Zhan Y, Chen Z, He S, Gong Y, He A, Li Y, Zhang L, Zhang X, Fang D, Li X, Zhou L. Long non-coding RNA SOX2OT promotes the stemness phenotype of bladder cancer cells by modulating SOX2. Mol Cancer. 2020;19:25.

17. Zhan Y, Lin J, Liu Y, Chen M, Chen X, Zhuang C, Liu L, Xu W, Chen Z, He A, et al. Up-regulation of long non-coding RNA PANDAR is associated with poor prognosis and promotes tumorigenesis in bladder cancer. J Exp Clin Cancer Res. 2016;35:83.

18. Zhan Y, Chen Z, Li Y, He A, He S, Gong Y, Li X, Zhou L. Long non-coding RNA DANCR promotes malignant phenotypes of bladder cancer cells by modulating the miR-149/MSI2 axis as a ceRNA. J Exp Clin Cancer Res. 2018;37:273.

19. Pan Z, Mao W, Bao Y, Zhang M, Su X, Xu X. The long noncoding RNA CASC9 regulates migration and invasion in esophageal cancer. Cancer Med. 2016:5:2442-7

20. Gao GD, Liu XY, Lin Y, Liu HF, Zhang GJ. LncRNA CASC9 promotes tumorigenesis by affecting EMT and predicts poor prognosis in esophageal squamous cell cancer. Eur Rev Med Pharmacol Sci. 2018;22:422-9.

21. Liang Y, Chen X, Wu Y, Li J, Zhang S, Wang K, Guan X, Yang K, Bai Y. LnCRNA CASC9 promotes esophageal squamous cell carcinoma metastasis through upregulating LAMC2 expression by interacting with the CREBbinding protein. Cell Death Differ. 2018;25:1980-95.

22. Klingenberg M, Gross M, Goyal A, Polycarpou-Schwarz M, Miersch T, Ernst AS, Leupold J, Patil N, Warnken U, Allgayer H, et al. The long noncoding RNA Cancer susceptibility 9 and RNA binding protein heterogeneous nuclear Ribonucleoprotein $L$ form a complex and Coregulate genes linked to AKT signaling. Hepatology. 2018;68:1817-32.

23. Yang $Y$, Chen D, Liu H, Yang K. Increased expression of IncRNA CASC9 promotes tumor progression by suppressing autophagy-mediated cell apoptosis via the AKT/mTOR pathway in oral squamous cell carcinoma. Cell Death Dis. 2019:10:41.

24. Wu Y, Hu L, Liang Y, Li J, Wang K, Chen X, Meng H, Guan X, Yang K, Bai Y. Up-regulation of IncRNA CASC9 promotes esophageal squamous cell carcinoma growth by negatively regulating PDCD4 expression through EZH2. Mol Cancer. 2017;16:150.

25. Luo K, Geng J, Zhang Q, Xu Y, Zhou X, Huang Z, Shi KQ, Pan C, Wu J. LncRNA CASC9 interacts with CPSF3 to regulate TGF-beta signaling in colorectal cancer. J Exp Clin Cancer Res. 2019;38:249.

26. Chen W, Zheng R, Baade PD, Zhang S, Zeng H, Bray F, Jemal A, Yu XQ, He J. Cancer statistics in China, 2015. CA Cancer J Clin. 2016;66:115-32.

27. Dinney CP, McConkey DJ, Millikan RE, Wu X, Bar-Eli M, Adam L, Kamat AM, Siefker-Radtke AO, Tuziak T, Sabichi AL, et al. Focus on bladder cancer. Cancer Cell. 2004;6:111-6.

28. Maia MC, Grivas P, Agarwal N, Pal SK. Circulating tumor DNA in bladder cancer: novel applications and future directions. Eur Urol. 2018;73:541-2

29. Antoni S, Ferlay J, Soerjomataram I, Znaor A, Jemal A, Bray F. Bladder cancer incidence and mortality: a global overview and recent trends. Eur Urol. 2017;71:96-108.

30. Roupret M. Words of Wisdom. Re: Finasteride reduces the risk of bladder cancer in a large prospective screening study. Eur Urol. 2016;69:537.

31. Boccardo F, Palmeri L. Adjuvant chemotherapy of bladder cancer. Ann Oncol. 2006;17(Suppl 5):v129-32.

32. Parisi S, Troiano M, Corsa P, Raguso A, Cossa S, Piazzolla EE, Munafo T, Sanpaolo G, Natuno A, Maiello E. Role of external radiation therapy in urinary cancers. Ann Oncol. 2007;18:vi157-61.

33. Meng L, Ward AJ, Chun S, Bennett CF, Beaudet AL, Rigo F. Towards a therapy for Angelman syndrome by targeting a long non-coding RNA. Nature. 2015;518:409-12.

34. Hon CC, Ramilowski JA, Harshbarger J, Bertin N, Rackham OJ, Gough J, Denisenko E, Schmeier S, Poulsen TM, Severin J, et al. An atlas of human long non-coding RNAs with accurate 5' ends. Nature. 2017;543:199-204.
35. Huan L, Guo T, Wu Y, Xu L, Huang S, Xu Y, Liang L, He X. Hypoxia induced LUCAT1/PTBP1 axis modulates cancer cell viability and chemotherapy response. Mol Cancer. 2020;19:11.

36. Li J, Wei L, Han Z, Chen Z, Zhang Q. Long non-coding RNA X-inactive specific transcript silencing ameliorates primary graft dysfunction following lung transplantation through microRNA-21-dependent mechanism. EBioMedicine. 2020;52:102600.

37. Chu C, Zhang QC, da Rocha ST, Flynn RA, Bharadwaj M, Calabrese JM, Magnuson T, Heard E, Chang HY. Systematic discovery of Xist RNA binding proteins. Cell. 2015;161:404-16.

38. Li S, Huang Y, Huang Y, Fu Y, Tang D, Kang R, Zhou R, Fan XG. The long non-coding RNA TP73-AS1 modulates HCC cell proliferation through miR200a-dependent HMGB1/RAGE regulation. J Exp Clin Cancer Res. 2017:36:51.

39. Su X, Li G, Liu W. The long noncoding RNA cancer susceptibility candidate 9 promotes nasopharyngeal carcinogenesis via stabilizing HIF1alpha. DNA Cell Biol. 2017;36:394-400.

40. Sassenberg M, Droop J, Schulz WA, Dietrich D, Loick SM, Wiek C, Scheckenbach K, Gaisa NT, Hoffmann MJ. Upregulation of the long noncoding RNA CASC9 as a biomarker for squamous cell carcinoma. BMC Cancer. 2019:19:806

\section{Publisher's Note}

Springer Nature remains neutral with regard to jurisdictional claims in published maps and institutional affiliations.

\section{Ready to submit your research? Choose BMC and benefit from:}

- fast, convenient online submission

- thorough peer review by experienced researchers in your field

- rapid publication on acceptance

- support for research data, including large and complex data types

- gold Open Access which fosters wider collaboration and increased citations

- maximum visibility for your research: over $100 \mathrm{M}$ website views per year

At $\mathrm{BMC}$, research is always in progress.

Learn more biomedcentral.com/submissions 